\title{
Redundancy phenomena are affected by response requirements
}

\author{
G. ROBERT GRICE and LYN CANHAM \\ University of New Mexico, Albuquerque, New Mexico
}

\begin{abstract}
Results are reported for two go/no-go reaction time (RT) experiments, in which the redundant targets advantage was investigated. These experiments were replications of two earlier choice reaction time (CRT) experiments, in which letter stimuli were used. Important differences between the go/no-go RT experiments and the CRT experiments were obtained. Equal and significant redundancy advantages were obtained whether redundant targets were compared with a single target presented with a noise letter or without noise. In the CRT experiments, the advantage was not obtained in the comparison with a single target presented alone. Noise letters did not slow the RTs to single targets with which they were presented as was the case with CRT. Since the differing results of the two procedures depend on the response requirements, explanation of differing CRT data in terms of perceptual or attentional concepts is probably inappropriate. The presence and absence of response competition in the two situations may be the best interpretation. The results tend to support a conclusion of the parallel processing of two letter stimuli separated spatially by as much as $3^{\circ}$.
\end{abstract}

In typical experiments, a redundancy gain refers to an improvement in accuracy in a recognition experiment or to a decrease in latency in a reaction time (RT) experiment when the target is presented more than once. Much of this literature has recently been reviewed by $\mathrm{C}$. W. Eriksen, Goettl, St. James, and Fournier (1989). In recognition experiments, the preponderance of the evidence indicates improved recognition with multiple presentation of the target. Results of the RT experiments are somewhat more complex. However, RT experiments are regarded as more relevant to current problems of interest in the area of information processing. In particular, these problems include singular versus parallel processing of stimuli and separate activation versus coactivation in response activation.

A number of the RT experiments in this area have been choice reaction time (CRT) experiments in which letters were used as stimuli. Generally, there have been two stimuli, each requiring a different response. The main results of several of these experiments (C. W. Eriksen et al., 1989; Fournier \& Eriksen, in press; Grice, Canham, \& Boroughs, 1984; Grice, Canham, \& Gwynne, 1984; Grice \& Gwynne, 1987) present a rather consistent pattern. There is a redundant signals advantage when the redundant signals condition is compared with a single target presented with an irrelevant, noise letter. It has been suggested by Grice, Canham, and Gwynne (1984) that this may not be a genuine redundancy effect but rather

This research was supported by Public Health Service Grant MH16400 from the Institute of Mental Health. Requests for reprints should be sent to G. Robert Grice, Department of Psychology, University of New Mexico, Albuquerque, NM 87131. the result of a distraction effect of the noise letter. Fournier and Eriksen (in press) suggest that this kind of redundant targets advantage is due to a singular focus of attention, which on about half of the trials will initially be directed to the noise letter rather than the target. On such trials, attention must be redirected to the target, and this requires time. With two targets present, the initial focus is always on a target, and no shift is necessary.

When the redundant targets condition is compared with single targets presented alone without noise, there is no evidence of a redundant targets advantage. Responses in the single-target condition are sometimes actually faster than they are with two targets. Grice, Canham, and Gwynne (1984) suggest that there may be some distraction when two stimuli are presented together, even though both are targets. This may suppress the possibility of a redundancy advantage and even result in faster responses to single targets. C. W. Eriksen et al. (1989) indicate that if attention is focused on one target in a singular fashion, no redundancy advantage would be expected with two targets. They further suggest that different results might be obtained with a closer spacing of stimuli.

In contrast with the results of the CRT experiments, the results with the go/no-go RT procedure are different. ${ }^{1}$ In this procedure, the subject is instructed to respond to one target only, and to withhold response to other stimuli. Most notably, van der Heijden, Schreuder, Maris, and Neerincx (1984) have obtained a significant redundant targets advantage with this procedure, when a two-target condition was compared with the mean of single targets in the faster stimulus position of each subject. ${ }^{2}$ The reason given for the use of this procedure was "to avoid response-competition problems." The authors did not use a noise letter with single targets in order to avoid distrac- 
tion effects. Further evidence of a difference between this and the CRT procedure is a comparison with a CRT experiment by Grice and Gwynne (1987). They failed to find a redundant targets advantage when the two-target condition was compared with the mean of the faster positions for each subject, in a situation in which the subjects never experienced noise letters.

Recently, Egeth and Mordkoff (in press), in an experiment that combined letter identity with a color dimension, also used the go/no-go RT procedure. They also found a redundant signals effect that was strong enough to satisfy Miller's (1982) criterion for rejecting serial and separate activation theories. This led them to the conclusion that the separable dimensions of shape and color could be processed in parallel. Of more immediate concern here is the finding, in a second experiment, that the presence or absence of a distractor had no effect. This certainly would not be expected on the basis of CRT data.

The reported differences in outcome between CRT and the go/no-go procedures could be due to experimental details other than the differences between the tasks. For example, the letter stimuli used by van der Heijden et al. (1984) may have been more discriminable than those used in the CRT experiments. However, if there are important differences between the procedures with respect to the outcomes obtained, it is important that we know this and attempt to understand the differences. If outcomes that we have attributed to perceptual or attentional factors are the result of different response requirements, some rethinking is in order. As a step toward clarifying this problem, we have replicated two of our CRT experiments, using the go/no-go RT procedure. All stimuli and combinations of experimental treatments are the same, except for the difference between the two procedures. This makes possible a comparison between the two methods.

\section{EXPERIMENT 1}

The first experiment was a go/no-go replication of a CRT experiment reported by Grice and Gwynne (1987, Experiment 5). The experiment included two groups of subjects, each presented with equal numbers of singletarget and two-target trials. For one group, the single targets were presented alone, and for the other, the nontarget position was filled with an irrelevant, noise letter. In other words, these experiments represented a betweensubjects manipulation of the presence and absence of noise on single-target trials.

\section{Method}

The stimuli and method of presentation were as described by Grice and Gwynne (1987) and by Grice, Canham, and Boroughs (1984). The letters were light on a dark background. They were approximately $.29^{\circ}$ wide and $.35^{\circ}$ high. The two letter positions were separated vertically by $3^{\circ}$, center to center. The fixation point was a plus sign halfway between the two letter positions. It was on at all times except while the letters were displayed. Two target letters were used, $H$ and $S$. Half of the subjects were instructed to respond as quickly as possible to $\mathrm{H}$ without making errors, and not to respond to $S$. The other half of the subjects responded to $S$. Responses were made by pressing a telegraph key with the index finger of the preferred hand. When an irrelevant letter was used in the nontarget position, it was the letter $\mathrm{Y}$. One group received the $\mathrm{Y}$ on all single-target trials, and the other group never received it. For both groups there were equal numbers of presentations of $\mathrm{H}$ and $\mathrm{S}$. On single-target trials, the target was equally often in the upper and lower positions. All subjects received the same irregular order of trials. There were a total of 300 trials. The first 50 were practice trials, which were followed by a brief rest. The remaining trials followed without interruption, with the first 10 of them treated as practice. Data were based on the final 240 trials, 120 of which were response trials. There were 40 trials with a single target in each position and 40 with two targets. The same numbers applied to the letter to which the subject was instructed not to respond.

There were $4 \mathrm{sec}$ between trials. The exposure duration of the letter stimuli was $150 \mathrm{msec}$. Each trial was preceded by $1 \mathrm{sec}$ by an auditory warning signal. The subjects were instructed to look at the fixation point at the signal and to be prepared to respond.

The subjects were undergraduate women from a course in introductory psychology; they received course credit for participation. This is the same population that was used by Grice and Gwynne (1987) in the CRT experiment. There were 28 subjects in each of the two groups.

\section{Results}

The mean RTs for Experiment 1 are presented in the first two columns of Table 1. For single targets, we have presented the means based on the averages of the two stimulus positions and the means of the faster position for each subject. The false alarm rate for the experiment was .013 .

The mean of the subjects' faster positions is generally favored for evaluating a redundant targets advantage. The reason for this is that it eliminates the simple explanation that, with two targets, there is always a target in the subject's favored position. For the present data, no difference in conclusion would result from the choice of means to evaluate. For the means of faster positions, the redundant targets advantage is observed for both the no-noise and the noise conditions. For the difference between one and two targets with no noise, $t(27)=3.67, p<.01$. For the noise condition, $t(27)=4.70, p<.001$. For the two conditions together, $F(1,54)=30.68, p<.001$. The

Table 1

Means and Standard Deviations of Reaction Times for Experiment 1 and the Equivalent Choice Reaction Time Experiment

\begin{tabular}{|c|c|c|c|c|}
\hline \multirow[b]{2}{*}{ Condition } & \multicolumn{2}{|c|}{ Experiment 1} & \multicolumn{2}{|c|}{ Choice Reaction Time } \\
\hline & $M$ & $S D$ & $M$ & $S D$ \\
\hline \multicolumn{5}{|c|}{ No Noise } \\
\hline Single targets & 343 & 24 & 443 & 68 \\
\hline Faster single targets & 334 & 23 & 434 & 66 \\
\hline Two targets & 321 & 26 & 433 & 68 \\
\hline \multicolumn{5}{|c|}{ With Noise } \\
\hline Single targets & 353 & 42 & 434 & 50 \\
\hline Faster single targets & 339 & 40 & 420 & 46 \\
\hline Two targets & 329 & 38 & 405 & 40 \\
\hline
\end{tabular}

*See Grice and Gwynne (1987, Experiment 5). All values are in milliseconds. 
interaction between the redundancy gain and the noise effect is not significant $[F(1,54)<1]$. This means that the presence of noise had no significant effect on the redundancy gain. In other words, there was no evidence of distraction produced by the noise.

Corresponding data from the equivalent CRT experiment are presented in the third and fourth columns of Table 1. A comparison of the data for the faster positions clearly indicates the differences in outcome. With no noise, there was a redundant targets advantage for the go/no-go experiment, but there is no such evidence in the CRT experiment. For single targets with noise, the redundancy advantage was obtained in both experiments. Since the two experiments were conducted with the same subject population, we believe that a statistical comparison is informative. In an analysis of the two experiments, there were two significant effects. As would be expected, the go/no-go experiment was faster than the CRT experiment $[F(1,108)=117.73, p<.001]$. The three-way interaction of methods $\times$ noise $\times$ number of targets was also significant $[F(1,108)=5.73, p<.025]$. This means that the dependency of the redundancy effect on the presence or absence of noise differs significantly between the two kinds of experiments. This confirms the conclusion based on inspection of the means.

For this experiment, we have conducted Miller's (1982) test, based on cumulative distribution functions (CDFs), which was proposed to distinguish between coactivation and separate activation interpretations of a redundant targets advantage. Thus, the two-target CDFs for each subject were computed at the .05 quantiles. These quantiles were also determined for the sums of the single-target probabilities. According to Miller's test, the two-target CDF can never precede the CDF for the sum of the single targets according to separate activation models. This condition did exist for the no-noise experiment at the .05 quantile. However, the two-target quantile preceded the single-target sum by only $5 \mathrm{msec}$. In spite of the small difference, it was rather consistent, in that 20 of the 28 subjects had a difference in this direction. This is a significant value for the sign test $(p<.05)$. However, the $t$ test of this small difference is not significant $[t(27)=$ $1.58, n . s$.$] . In view of the small difference, no great im-$ portance should be attached to this result. On the other hand, in view of the conservative nature of Miller's test, supporters of separate activation theory should take no great comfort from the result. In the case of the noise condition, the .05 quantile for the sum of the single targets preceded that for both stimuli by $4 \mathrm{msec}$, so Miller's test was not applicable. This does not mean that coactivation theory must be rejected and separate activation theory accepted.

\section{EXPERIMENT 2}

The second experiment is a go/no-go replication of a CRT experiment by Grice, Canham, and Gwynne (1984, Experiment 2). This experiment involves a within-subjects manipulation of the presence and absence of noise on single-target trials. There is a single group of subjects, with three experimental conditions: single targets alone, single targets with the noise letter in the nontarget position, and targets in both positions.

\section{Method}

The stimuli, method of presentation, number of trials, and other procedures were the same as in Experiment 1. However, the 120 response trials consisted of $\mathbf{4 0}$ single-target trials with no noise, 40 single-target trials with the letter $Y$ in the nontarget position, and 40 trials with target in both positions. For single-target trials, the targets were in the upper and lower positions equally often. Everything except the go/no-go procedure was the same as it was in the CRT experiment of Grice, Canham, and Gwynne (1984). The subjects were 28 women from the same population that was used in the other experiments.

\section{Results}

Mean RTs for the three experimental conditions of the second experiment are presented in the first two columns of Table 2. Again we have presented, for single targets, both the means of both positions and the means of the subjects' faster positions. Statistical analyses are based on the faster positions. The false alarm rate to the nontarget stimuli was .019 .

When the single-target conditions are compared with the two-target condition, significant redundancy gains are observed in both instances. For the no-noise condition, $t(27)=5.17, p<.001$. For the condition with noise, $t(27)=3.23, p<.01$. It is clear that the noise letter paired with the single target did not produce a distraction or interference effect. The small difference between the two single-target conditions is actually in the opposite direction. However, the difference is not significant $[t(27)$ $=1.17$, n.s.].

Data from the corresponding CRT experiment are also presented in the third and fourth columns of Table 2. Certainly, there is no redundancy advantage for the no-noise condition, for the difference is significantly in the wrong direction $[t(27)=3.88, p<.001]$. When the redundancy effects are compared for the no-noise conditions across the two kinds of experiments, a highly significant methods

Table 2

Means and Standard Deviations of Reaction Times for Experiment 2 and the Equivalent Choice Reaction Time Experiment

\begin{tabular}{|c|c|c|c|c|}
\hline \multirow[b]{2}{*}{ Condition } & \multicolumn{2}{|c|}{ Experiment 2} & \multicolumn{2}{|c|}{ Choice Reaction Time* } \\
\hline & $M$ & $S D$ & $M$ & $S D$ \\
\hline \multicolumn{5}{|c|}{ No Noise } \\
\hline Single target & 353 & 36 & 435 & 67 \\
\hline Faster single target & 340 & 34 & 427 & 65 \\
\hline \multicolumn{5}{|c|}{ With Noise } \\
\hline Single target & 354 & 40 & 469 & 80 \\
\hline Faster single target & 336 & 38 & 458 & 78 \\
\hline Two targets & 324 & 40 & 437 & 69 \\
\hline
\end{tabular}

*See Grice, Canham, and Gwynne (1984, Experiment 2). All values are in milliseconds. 
$\times$ number of targets interaction is obtained $[F(1,54)=$ $41.66, p<.001$ ]. It can also be seen that the single target with noise is much slower than that without noise in the CRT experiment, but this is not true in the go/no-go experiment. When single targets with and without noise are compared for the two experiments, the noise $x$ methods interaction is significant $[F(1,54)=27.31$, $p<.001]$. The evidence is strong that noise impairs response to a single target in the CRT experiment but not with the go/no-go procedure. It is also clear that the redundant target advantage occurs with no noise with the go/nogo procedure but not with CRT.

It is also of interest to compare Experiments 1 and 2 in order to examine the extent to which the manner of manipulating noise affects the outcome. When single targets without noise are compared with two targets for the two experiments, the joint effect of number of targets is significant $[F(1,54)=38.18, p<.001]$. The experiments $\times$ number of targets interaction is not significant $[F(1,54)<1]$. A similar comparison for single targets with noise with two targets yields comparable results. For the joint redundancy effect, $F(1,54=26.67, p<.001$. The number of targets $\times$ experiments interaction is not significant $[F(1,54<1]$. In other words, the two experiments have produced highly consistent results.

\section{GENERAL DISCUSSION}

The go/no-go experiments reported here have shown two important differences from the results of comparable CRT experiments. First, a redundant targets advantage was obtained with the go/no-go procedure when two targets were compared with single targets without a noise letter in the nontarget position. This was not obtained in the CRT experiments. Second, a noise letter did not slow response to a target with which it was presented, as was the case in the CRT experiments. These differences apply not only to the CRT experiments that were replicated here with the go/no-go procedure, but also to other comparable experiments in the literature. The findings raise some fairly obvious problems for previous interpretations of CRT data that have been expressed in terms of purely perceptual or attentional processes. For example, Grice, Canham, and Boroughs (1984) and Grice, Canham, and Gwynne (1984) attributed the redundant targets effect, observed when noise letters were present, to a distracting effect of the noise letter. They further attributed the lack of a redundancy effect with no noise, as well as the faster response to a single target, to distraction. They suggested that when two letters are presented, even though both are targets, some level of distraction will occur. This would tend to slow response to two targets. A smaller effect of distraction would be produced than would be obtained with a nonidentical noise letter. However, distraction is a perceptual concept, and it would not make much sense to suggest that it would be present or absent merely as the result of different response requirements.
Fournier and Eriksen (in press) have interpreted the superiority of a two-target presentation over a single target with noise in terms of attention. They suggest that attention is focused on a single letter at a time. In the case of a noise letter with a target, the first fixation of attention will be on the noise letter about half of the time. Thus, in order to identify the target, the letters must be inspected serially when the initial focus is on noise. Time is required to shift the focus of attention. The RT difference between two targets and a single target with noise was within the range of RTs in other experiments with a similar interpretation. One basis of evidence for the singular focus was that there was no evidence for interference when two opposing targets were presented simultaneously. The separation between the two letters in their experiment was about the same as that in the present experiments. We certainly agree that this was a reasonable interpretation of their data. It was supported by the usual finding in CRT experiments that no redundant targets advantage was obtained in comparison with single targets alone. However, such data were not obtained in our go/no-go experiments. It appears that whether focal attention is singular or parallel should depend on the nature of the display and the perceptual task but not on the response requirements. At best, the Fournier and Eriksen interpretation seems to be of limited generality.

Since some of the more promising of our explanations of CRT data in this area are now in trouble, it seems desirable to find another approach that will account for these data, and at the same time to account for the differences between the go/no-go and the CRT procedures. At least initially, we think that response competition is the most promising, since it is present in CRT but not in go/no-go experiments. The different applicability of the concept to the two procedures has some direct experimental support. Since the work of B. A. Eriksen and C. W. Eriksen (1974), it has been repeatedly confirmed that, when a centrally presented target letter is flanked by response-incompatible letters, interference will be produced. Using measurement of partial responses and muscle potentials, Coles, Gratton, Bashore, Eriksen, and Donchin (1985) found that partial errors were frequent on such trials, and that correct RTs were longer when these occurred. Grice, Canham, and Schafer (1982) conducted two go/no-go replications of some of their CRT experiments in which this interference effect had been obtained. They found no evidence of interference with the go/no-go procedure. They concluded that for one to obtain the competition effect, there must be two active competing responses. It is probably safe to state that in the CRT procedure there is always at least some tendency to make errors, whether they are made or not. It should not be surprising if some nonobvious conditions should produce some level of competition. For example, Coles et al. (1985) found some partial error responses even on trials in which the target was flanked by an identical letter.

With respect to a noise letter accompanying a target, there is direct evidence of competition when the noise let- 
ter is similar to the other target (C. W. Eriksen et al., 1989). It is probably not unreasonable to think that any letter different from the target will result in some level of competition, particularly on trials when the response criterion is low. Explanation of the failure to obtain the redundant targets advantage in these terms is not obvious for the no-noise comparison. However, in view of the ubiquitous nature of competition in CRT, together with the variability of response criteria, it is quite possible that there is sufficient response competition to mask the occurrence of the redundancy effect. On trials in which the criterion for the incorrect response is lower than that for the correct response, the onset of any stimulus may initiate an incipient error response. The inhibition of this response would delay the correct response. Such an effect may be exaggerated with the onset of two stimuli rather than one. Van der Heijden et al. (1984) expressed suspicion of response competition in CRT, even though they used no noise letters on single-target trials. We are suggesting that their suspicion was correct.

Concerning broader issues in information processing, our data suggest that letters are processed in parallel with a spatial separation as great as $3^{\circ}$. Concerning separate activation as opposed to coactivation, we regard the data as inconclusive. Perhaps there is a slight edge to coactivation.

\section{REFERENCES}

Coles, M. G. H., Gratton, G., Bashore, T. R., Eriksen, C. W., \& Donchin, E. (1985). A psychophysiological investigation of the continuous flow model of information processing. Journal of Experimental Psychology: Human Perception \& Performance, 11, 529-553.

EGETH, H. E., \& MORDKOFF, J. T. (in press). Redundancy gain revisited: Evidence for parallel processing of separable dimensions. In J. Pomer- antz \& G. Lockhead (Eds.), The perception of structure. Washington, DC: American Psychological Association.

ERIKSEN, B. A., \& ERIKSEN, C. W. (1974). Effects of noise letters upon the identification of a target letter in a nonsearch task. Perception \& Psychophysics, 16, 143-149.

Eriksen, C. W., Goettl, B., St. James, J. D., \& Fournier, L. R. (1989). Processing redundant signals: Coactivation, divided attention, or what? Perception \& Psychophysics, 45, 356-370.

Fournier, L. R., \& Eriksen, C. W. (in press). Coactivation in the perception of redundant targets. Journal of Experimental Psychology: Human Perception \& Performance.

Grice, G. R., Canham, L., \& Boroughs, J. M. (1984). Combination nule for redundant information in reaction time tasks with divided attention. Perception \& Psychophysics, 35, 451-463.

Grice, G. R., Canham, L., \& GWynNe, J. W. (1984). Absence of a redundant-signals effect in a reaction time task with divided attention. Perception \& Psychophysics, 36, 565-570.

Grice, G. R., Canham, L., \& SChafer, C. (1982). Role of the response in associative interference. Bulletin of the Psychonomic Society, 20, 214-216.

Grice, G. R., \& GWYNnE, J. W. (1987). Dependence of target redundancy effects on noise conditions and number of targets. Perception \& Psychophysics, 42, 29-36.

Miller, J. (1982). Divided attention: Evidence for coactivation with redundant signals. Cognitive Psychology, 14, 247-279.

van der Heijden, A. H. C., Schreuder, R., Maris, L., \& NeERINCX, M. (1984). Some evidence for correlated separate activation in a simple letter-detection task. Perception \& Psychophysics, $36,577-585$

\section{NOTES}

1. In previous reports from this laboratory, when we have used the go/no-go procedure (or c-reaction), we have referred to it as disjunctive reaction time (DRT). We have used go/no-go here, because, currently, it seems to be the most commonly used and best understood term.

2. Grice and Gwynne (1987) incorrectly stated that van der Heijden et al. (1984) did not compare the redundant targets condition with the mean of the subjects' faster positions. The senior author sincerely regrets this error.

(Manuscript received February 12, 1990; accepted for publication April 4, 1990.) 